\title{
Inferior Vena Cava Agenesis and Deep Vein Thrombosis: A Pharmacological Alternative to Vitamin K Antagonists
}

\author{
$\underline{\text { Inês Esteves } \text { Cruz }^{1} \text {, Pedro Ferreira }}{ }^{2}$, Raquel Silva ${ }^{1}$, Francisco Silva ${ }^{1}$, Isabel Madruga ${ }^{1}$ \\ ${ }^{1}$ Hospital Egas Moniz, Lisbon, Portugal \\ ${ }^{2}$ Hospital Santa Maria, Lisbon, Portugal
}

Received: 02/10/2019

Accepted: 08/10/2019

Published: 06/12/2019

\begin{abstract}
How to cite this article: Esteves Cruz I, Ferreira P, Silva R, Silva F, Madruga I. Inferior vena cava agenesis and deep vein thrombosis:a pharmacological alternative to vitamin K antagonists. EJCRIM 2019;6: doi:10.12890/2019_001310.
\end{abstract}

Conflicts of Interests: The Authors declare that there are no competing interest

This article is licensed under a Commons Attribution Non-Commercial 4.0 License

\section{ABSTRACT}

Inferior vena cava (IVC) agenesis is a rare congenital abnormality affecting the infrarenal segment, the suprarenal or the whole of the IVC. It has an estimated prevalence of up to $1 \%$ in the general population that can rise to $8.7 \%$ when abnormalities of the left renal vein are considered. Most IVC malformations are asymptomatic but may be associated with nonspecific symptoms or present as deep vein thrombosis (DVT). Up to $5 \%$ of young individuals under 30 years of age with unprovoked DVT are found to have this condition. Regarding the treatment of IVC agenesis-associated DVT, there are no standard guidelines. Treatment is directed towards preventing thrombosis or its recurrence. Low molecular weight heparin and oral anticoagulation medication, in particular vitamin K antagonists (VKAs) are the mainstay of therapy. Given the high risk of DVT recurrence in these patients, oral anticoagulation therapy is suggested to be pursued indefinitely. As far as we know, this is the first case reporting the use of a direct factor Xa inhibitor in IVC agenesis-associated DVT. Given VKA monitoring limitations, the use of a direct Xa inhibitor could be an alternative in young individuals with anatomical defects without thrombophilia, but further studies will be needed to confirm its efficacy and safety.

\section{LEARNING POINTS}

- Up to $5 \%$ of young individuals under 30 years of age with unprovoked deep vein thrombosis (DVT) are found to have this condition. Therefore, these types of anomalies should be actively looked for, particularly in young patients with DVT.

- Treatment with low molecular weight heparin or oral anticoagulation medication is the mainstay of therapy, directed towards preventing thrombosis or its recurrence.

- A direct factor Xa inhibitor could be a possible alternative to vitamin K antagonists in these patients, despite the lack of clinical evidence supporting its use at the moment.

\section{KEYWORDS}

Inferior vena cava agenesis, deep vein thrombosis, direct factor Xa inhibitors, rivaroxaban

\section{INTRODUCTION}

The inferior vena cava (IVC) is formed by the development, regression and anastomosis of 3 pairs of veins (posterior cardinal, subcardinal and supracardinal) between the sixth and eighth weeks of gestation. If this process is not completed in time or if intrauterine or perinatal thrombosis occurs, malformations can take place. These include a duplicated IVC, IVC agenesis or the interruption of a specific segment. Up to $5 \%$ of young individuals under 30 years of age with unprovoked DVT are found to have this condition ${ }^{[1]}$. Imaging criteria for IVC agenesis 
diagnosis include absence of the IVC lumen, associated with a connection between the existent caval segment and the azygous system, and the existence of venous collaterals (paravertebral venous system and its communications with the ascending lumbar veins and azygoushemiazygous system; gonadal, periureteral and other retroperitoneal veins; abdominal wall veins; haemorrhoidal venous plexus and the portal venous system $)^{[2]}$.

\section{CASE DESCRIPTION}

A 20-year-old male, with no relevant personal history, presented to the emergency department with lumbar and abdominal pain, that worsened with exertion and Valsalva manoeuvres, and an inability to walk. At examination, a pronounced bilateral leg oedema was observed. There were no other relevant abnormal findings including the abdominal exam. Blood test results were normal. A CT scan of the lumbar spine was performed that also showed no abnormalities. Finally, a venous duplex ultrasound scan showed bilateral deep venous thrombosis of both the femoral and external iliac veins.

Given the extent of the thrombosis in a young healthy male, an abdominal CT scan was performed to rule out further extension of thrombosis or a possible malignancy. It revealed agenesis of the infrarenal segment of the IVC (at the D10 level), with evidence of collateral circulation to ascending lumbar veins (Fig. 1). Furthermore, the left renal vein drained into the homolateral ascending lumbar vertebral vein and the right renal vein drained into the portal system by porto-renal anastomoses. Diffuse ectasia of the vena cava tributary venous return system, with multiple venous anastomoses, was also evident.
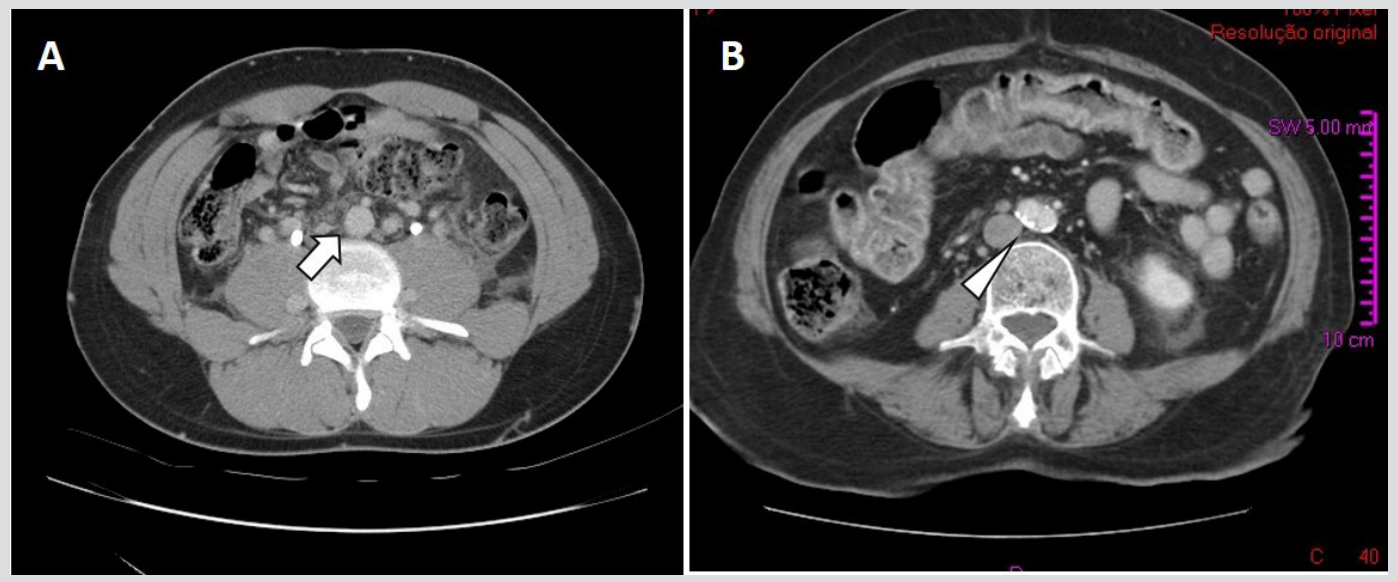

Figure 1. (A) IVC agenesis of the infrarenal segment (CT scan). The IVC is missing (arrow). (B) The corresponding axial image in a normal patient (arrowhead)

Due to IVC agenesis-associated DVT, treatment with low molecular weight heparin (LMWH) was initiated and changed to warfarin at the time of discharge. Follow-up at 12 months showed no progression of thrombosis and thrombophilia screening was normal (lupus anticoagulant, cardiolipin antibody, beta-2 glycoprotein IgG and IgM, antithrombin, protein C, protein S, factor V Leiden [G1691A] mutation, prothrombin gene [G20210A] mutation, homocysteine).

Around this time, faced with life-long anticoagulation therapy, the patient raised significant objections to warfarin use and enquired about alternatives. Fearing possible therapeutic non-compliance, treatment with rivaroxaban $20 \mathrm{mg} / \mathrm{day}$ was initiated, even though no clinical evidence exists to support the use of direct factor Xa inhibitors in this condition.

At the 3-year follow-up with rivaroxaban, there was no evidence of new thrombotic events. During this period, however, collateral circulation in the lower abdomen and lower limbs increased substantially (Fig. 2 and Fig. 3). Re-evaluation with a venous Doppler ultrasound scan continued to show signs of venous thrombosis in both the femoral and external iliac veins, with scarce recanalization. Due to the lack of significant recanalization, rivaroxaban was reduced to $10 \mathrm{mg} /$ day considering secondary prevention of DVT. No thrombotic events have been documented since rivaroxaban dose reduction a year ago.

\section{DISCUSSION}

Most patients with IVC agenesis are asymptomatic and the anomaly is usually detected incidentally. Deep venous thrombosis is the most common form of presentation and its risk is thought to be increased in patients with IVC anomalies. Therefore, these types of anomalies should be actively looked for, particularly in young patients with DVT. 
Other less frequent presentations include sciatic neuropathy, caused by dilated epidural veins compressing lumbar nerve roots, obstructive pyelonephritis, pelvic congestion syndrome in women and varicocele in men $^{[3]}$.

These anomalies lead to an estimated 5-9.5\% of unprovoked DVT in patients < 30 years of age, probably due to an inadequate return of blood, despite prominent collaterals. This in turn may lead to venous stasis and subsequent DVT. DVT is bilateral in more than $50 \%$ of patients with IVC agenesis, in contrast to less than $10 \%$ of patients with a normal IVC ${ }^{[2]}$. Pulmonary embolism in these patients is an uncommon finding (9.67\%), as emboli are trapped in the azygous-hemiazygous system before reaching the pulmonary circulation.

A precipitant factor has been reported in multiple cases of acute thrombosis in patients with IVC agenesis, most notably, strenuous muscular exercise (up to $20.6 \%$ of cases) ${ }^{[2]}$. Sufficient blood flow during increased physical exertion is probably not supported by the existing collaterals, and consequently, venous stasis and clotting ensue. Other factors associated with an inherited or acquired thrombotic risk should still be sought and a thrombophilia screening is recommended. Acquired factors include immobilization, trauma, surgery, vascular catheterization, smoking or the use of oral contraceptives.

In addition to being able to identify an acute DVT, a lower extremity venous duplex ultrasound scan may show direct or indirect signs of chronic central venous obstruction, such as damaged valves and significant reflux in peripheral deep veins, as well as acute central venous obstruction through a diminished or absent respiratory flow phasicity. Significant superficial collateral networks emanating from the abdominal wall or the pelvis can also be identified by a duplex ultrasound scan. CT venography or magnetic resonance venography are usually necessary to diagnose and determine the exact anatomic variant of congenital IVC agenesis.

Regarding the treatment of IVC agenesis-associated DVT, there are no standard guidelines. Treatment is directed towards preventing thrombosis or its recurrence. LMWH and oral anticoagulation medication, in particular vitamin K antagonists (VKAs), in combination with pressotherapy and leg elevation, are the mainstays of therapy ${ }^{[4]}$. No precise duration of anticoagulation therapy has been established. In a literature review of 72 cases, Lambert et al. showed that for $77.8 \%$ of patients, a VKA was prescribed without information about the total duration of therapy ${ }^{[1]}$. However, given the high risk of DVT recurrence in these patients, oral anticoagulation therapy is suggested to be pursued indefinitely, with frequent re-evaluations due to the associated haemorrhagic risk.

As far as we know, this is the first case reporting the use of a direct factor Xa inhibitor in IVC agenesis-associated DVT. Given VKA monitoring limitations, the use of a direct Xa inhibitor could be an alternative in young individuals with anatomical defects without thrombophilia, but further studies will be needed to confirm its efficacy and safety. In our case, there was no evidence of DVT recurrence at the 3-year follow- 
up with rivaroxaban $20 \mathrm{mg} / \mathrm{day}$. Based on the EINSTEIN CHOICE trial[5], anticoagulation therapy was lowered to $10 \mathrm{mg} / \mathrm{day}$ to account for secondary prevention of DVT. At the 12-month follow-up there was no evidence of new thrombotic events.

At the moment, there are also other therapeutic options to consider, such as catheter-directed thrombolysis (CDT) for fast thrombus removal in patients with IVC agenesis with acute DVT, particularly iliofemoral venous thrombosis. Broholm et al. argued that CDT provides immediate symptom relief and significantly decreases thrombus burden that may otherwise take days to weeks to resolve with systemic anticoagulation therapy alone ${ }^{[6]}$. Pharmacomechanical CDT, a combination of CDT and mechanical thrombectomy, also seems to be yielding promising results. Compared to systemic anticoagulation therapy alone, it has been shown to significantly decrease the thrombus burden, the incidence of recurrent DVT and the incidence of post-thrombotic syndrome. However, more research is required before definite conclusions can be drawn. Patients should also be advised to avoid additional risk factors such as prolonged immobilization and oral contraceptive use. In conclusion, the investigation of idiopathic DVT in a young person should include a hypercoagulability evaluation as well as an imaging examination looking for congenital abnormalities of the deep venous system, including IVC agenesis. Other circumstances, such as a severe bilateral lower extremity chronic venous insufficiency or extensive bilateral iliofemoral thrombosis in a young patient, should also prompt a thorough imaging assessment. In our case, the use of a direct factor Xa inhibitor in a patient with IVC agenesis appeared to be safe, with no evidence of DVT recurrence after 4 years of follow-up. Thus, a direct factor Xa inhibitor could be a possible alternative to VKAs in these patients, despite the lack of clinical evidence supporting its use at the moment.

\section{REFERENCES}

1. Gensas C, Pires L, Kruse M, Leiria T, Gomes D, Lima G. Agenesis of the inferior vena cava. Rev Bras Cardiol Invasiva 2012;20(4):427-430

2. Lambert M, Marboeuf P, Midulla M, Trillot N, Beregi JP, Mounier-Vehier C. Inferior vena cava agenesis and deep vein thrombosis: 10 patients and review of the literature. VasC Med 2010;15:451-459.

3. Singh S, Bhatt T. Inferior vena cava agenesis: a rare cause of pelvic congestion syndrome. J Clin Diagn Res 2017;11(3):06-08.

4. Kalogridaki E, Kanioura E, Mavrigiannaki E, Katralis P, Filippou D, Skandalakis P, et al. Deep vein thrombosis and inferior vena cava agenesis. J Pediatr Surg Case Rep 2018;34:1-3.

5. Weitz JI, Lensing AWA, Prins MH, Bauersachs R, Beyer-Westendorf J, Bounameaux H, et al. Rivaroxaban or aspirin for extended treatment of venous thromboembolism. N Engl J Med 2017;376:1211-1222.

6. Broholm R, Jorgensen M, Just S, Jensen LP, Baekgaard N. Acute iliofemoral venous thrombosis in patients with atresia of the inferior vena cava can be treated successfully with catheter directed thrombolysis. J Vasc Interv Radiol 2011;22(6):801-805. 